\title{
Female offspring born to obese and insulin resistant dams are not at increased risk for obesity and metabolic dysfunction during early development
}

\begin{tabular}{|r|l|}
\hline Journal: & Canadian Journal of Physiology and Pharmacology \\
\hline Manuscript ID & cjpp-2017-0371.R2 \\
\hline Danuscript Type: & Brief Report \\
\hline Complete List of Authors: & $\begin{array}{l}\text { Aburasayn, Hanin; University of Alberta, Pharmacy and pharmaceutical } \\
\text { science } \\
\text { Al Batran, Rami; University of Alberta } \\
\text { Gopal, Keshav; University of Alberta } \\
\text { Almutairi, Malak; University of Alberta } \\
\text { Eshreif, Amina; University of Alberta } \\
\text { Eaton, Farah; University of Alberta } \\
\text { Ussher, John; University of Alberta, }\end{array}$ \\
\hline Is the invited manuscript for & N/A \\
consideration in a Special \\
Issue?:
\end{tabular}


1 Female offspring born to obese and insulin resistant dams are not at increased risk for

2

$11{ }^{\dagger}$ Address for correspondence:

12 Dr. John R. Ussher,

$132-020 \mathrm{C}$

14 Katz Centre for Pharmacy and Health Research

15 University of Alberta

16 Edmonton, AB T6G 2E1

17 Canada

tel: $1-780-492-9337$

fax: 1-780-492-3175

21

email: jussher@ualberta.ca

22

23 Running Title: Obese dams and metabolic health of female offspring

24 Word Count: 2,986

25 Figures: 4
${ }^{1}$ Faculty of Pharmacy and Pharmaceutical Sciences, University of Alberta, Edmonton, AB Canada

${ }^{2}$ Alberta Diabetes Institute, University of Alberta, Edmonton, AB Canada

${ }^{3}$ Women and Children's Health Research Institute, University of Alberta, Edmonton, AB Canada 


\section{Abstract:}

27 The percentage of women who are obese at the time of conception/pregnancy is increasing, with 28 animal and human studies demonstrating that offspring born to obese dams/mothers are at increased 29 risk for obesity and the metabolic syndrome. Our goal was to confirm in an experimental model of 30 metabolic syndrome in the dam, whether the offspring would be at increased risk of obesity. 31 Conversely, we observed that male offspring born to dams with metabolic syndrome had no 32 alterations in their body weight profiles, whereas female offspring born to dams with metabolic 33 syndrome were heavier at weaning, but exhibited no perturbations in energy metabolism. Moreover, 34 they gained weight at a reduced rate versus female offspring born to healthy dams, and thus weighed 35 less at study completion. Hence, our findings suggest that factors other than increased 36 adiposity/insulin resistance during pregnancy are responsible for the increased risk of obesity in 37 children born to obese mothers.

Key Words: Obesity, pregnancy, high fat diet, offspring, glucose homeostasis, metabolic syndrome 


\section{Introduction}

Currently in Canada there are over 2 million children who are overweight/obese, and the majority of these children will grow up to become adults who are also obese and at increased risk for developing metabolic syndrome (Morrison et al. 2007). Of interest, the concept of "developmental programming" suggests that an individual's risk for developing disease throughout life is intricately connected to the environment of the womb that individual was exposed to (Blackmore and Ozanne 2013). This idea was first put forward by David Barker and colleagues and termed the "fetal origins hypothesis" to explain the inverse relationship between birth weight and mortality resulting from ischemic heart disease (Barker et al. 1989). Over the years the "fetal origins hypothesis" has continued to evolve, with a more recent evolution of this initial concept termed the "Developmental Origins of Health and Disease (DOHaD)". The DOHaD hypothesis postulates that the risk for disease susceptibility in a developing individual is not simply confined to prenatal and early postnatal development, but is actually influenced by a more broader developmental window that includes the period from pre-conception to fertilization of the oocyte (Blackmore and Ozanne 2013; Pereira et al. 2015).

With regards to childhood obesity, as there are now an increasing percentage of pregnancies in overweight/obese women at conception (Flegal et al. 2012), the DOHaD hypothesis would suggest that this may be a significant factor accounting for the increased rates of obesity in our pediatric population (Catalano 2003; Shankar et al. 2008). Indeed, a number of studies have demonstrated that offspring born to obese dams are at increased risk for obesity and obesity-related metabolic dysfunction, with many studies implicating a key role for epigenetic alterations in mediating this phenomenon (Borengasser et al. 2013; Laker et al. 2014). For example, Howie and colleagues demonstrated that offspring born to dams supplemented with a high-fat diet (HFD) prior to and during pregnancy/lactation are more susceptible to obesity, regardless if they are weaned onto a low-fat diet (LFD) or HFD (Howie et al. 2009). Moreover, it has been demonstrated that C57BL/6J mice fed a HFD prior to and during pregnancy/lactation produce offspring at increased risk of glucose intolerance, 
which was associated with reduced peroxisome proliferator-activated receptor gamma coactivator 1alpha (PGC1 $\alpha$ ) mRNA expression as a result of increased PGC1 $\alpha$ gene methylation (Laker et al. 2014). Nevertheless, there is inconsistency among studies exploring maternal obesity's actions on offspring development, as some studies have observed no impact of maternal obesity on offspring body weight (Buckley et al. 2005; Holemans et al. 2004).

Therefore, our objective was to develop an animal model of obesity and related metabolic dysfunction in the pregnant dam to study whether the DOHaD hypothesis would be relevant to and impact the developing offspring. If this proved to be correct, our secondary objective was to assess whether such findings may be due to altered expression of critical genes regulating energy metabolism.

\section{Methods}

\section{Animal Care}

All animals received care according to the Canadian Council on Animal Care and the University of Alberta Health Sciences Animal Welfare Committee. 8-week-old C57BL/6J (Jackson Laboratory) females were either fed a low-fat diet (LFD, 10\% kcal from lard, Research Diets D12450J) or high-fat diet (HFD, 60\% kcal from lard, Research Diets D12492) for 5 weeks. After 5 weeks of LFD/HFD supplementation, females were mated to a 12-week-old male C57BL/6J mouse supplemented with a LFD. Upon confirmation of a vaginal plug, the male breeder was removed and female dams remained on their respective diet throughout gestation. Following birth, pups born to the dam supplemented with a LFD remained with the dam throughout the 21-day nursing/lactation period, whereas offspring born to the dam supplemented with a HFD were cross-fostered to a surrogate dam supplemented with a LFD. This was carried out to ensure that potential body weight differences would not be due to offspring being nursed by an insulin resistant dam receiving their nutrition primarily from a HFD. A total of 4 dams were used for each experimental group ( 4 surrogate dams to foster pups from the 4 dams supplemented with a HFD, and 4 dams supplemented with a LFD). For each individual breeding, it 
94 was ensured that litter sizes between the surrogate dam and the LFD dam were identical at 3 days 95 post-birth, with a maximum litter size of 5 . Hence, if one dam was nursing 5 pups at 3 days post-birth 96 and another dam was nursing 4 pups, 1 pup was randomly euthanized from the dam nursing 5 pups, 97 such that both dams nursed an identical number of pups (4 pups) from 3 days post-birth until weaning. 98 At 21-days of age all offspring were weaned into separate cages and supplemented with a LFD until 99 euthanization at $14-$ weeks of age.

100

Magnetic Resonance Imaging (MRI) Body Composition Analysis

102 All female dams and offspring underwent assessment of body composition via quantitative nuclear 103 magnetic resonance relaxometry to quantify total lean/fat mass utilizing an EchoMRI-4in1/700 body 104 composition analyzer.

105

\section{Glycemic Assessments}

107 We assessed glucose homeostasis in female offspring as previously described (Ussher et al. 2014). In brief, oral and intraperitoneal glucose tolerance were assessed either in female dams fed a LFD or HFD for 5-weeks, or in female offspring following an overnight fast at 10- and 11-weeks of age, respectively, using a glucose dose of $2 \mathrm{~g} / \mathrm{kg}$ body weight. Intraperitoneal insulin tolerance was assessed either in female dams fed a LFD or HFD for 5-weeks, or in female offspring following a $6 \mathrm{hr}$

112 fast at 12-weeks of age, using an insulin dose of $0.3 \mathrm{U} / \mathrm{kg}$ body weight (Novolin (biosynthetic human 113 insulin), Novo Nordisk).

Indirect Calorimetry

116 In vivo metabolic assessment via indirect calorimetry was performed in female offspring at 8-weeks of 117 age using an Oxymax comprehensive lab animal monitoring system (Columbus Instruments) as 118 previously described (Ussher et al. 2016). 
Statistical Analysis

121 All values are presented as means \pm standard error of the mean (SEM). Significant differences were

122 determined by the use of an unpaired, two-tailed Student's $t$-test, or a two-way analysis of variance 123 (ANOVA) followed by a Bonferroni post-hoc analysis.

\section{Results}

126 5-weeks of high fat feeding leads to a marked increase in adiposity and impairs glucose 127 homeostasis in female dams.

8-week-old female C57BL/6J mice were fed either a LFD or HFD for 5 weeks prior to conception with a lean 12-week-old male C57BL/6J mice fed a LFD. 5-weeks of HFD supplementation lead to significant increases in body weight in female mice, and this was associated with a significant increase

131 in total fat mass and percent body fat (Figure 1A-C). This increase in adiposity was associated with impaired glucose homeostasis in female mice fed the HFD, as they exhibited both worse glucose and insulin tolerance versus their female counterparts fed a LFD (Figure 1D/E).

Female offspring born to dams supplemented with a HFD are heavier at weaning but exhibit reductions in weight gain throughout early development. to their counterparts born to dams fed a LFD, while also gaining weight at a similar rate (Figure 2).

139 Conversely, female offspring born to dams fed a HFD for 5 weeks prior to conception were 140 significantly heavier at weaning than their counterparts born to dams fed a LFD (Figure 3A). This 141 difference in body weight quickly dissipated by 6- to 8-weeks of age, as female offspring born to dams 142 fed a LFD gained body weight at an accelerated pace (Figure 3B). At study completion when the 143 female offspring were 14-weeks of age, those born to a dam fed a HFD actually weighed less than 144 those born to a dam fed a LFD (Figure 3A). These differences in body weight at weaning and at study 
145 completion in female offspring were not associated with changes in total fat mass and percent body fat 146 (Figure 3C-E).

Glucose homeostasis and in vivo energy metabolism are normal in female offspring born to dams supplemented with a HFD.

Because the male offspring demonstrated similar body weight curves throughout their early development (Figure 2), we focused the rest of our studies on the female offspring. When all female offspring were 10- and 11-weeks of age, they underwent oral and intraperitoneal glucose tolerance tests, respectively, and we observed no differences in glucose tolerance between both groups of offspring (Figure 4A/B). In addition, the female offspring born to dams fed a HFD exhibited no abnormalities in insulin sensitivity at 12-weeks of age in response to an insulin tolerance test (Figure 4C). During assessment of indirect calorimetry, we observed no differences in whole body oxygen consumption rates normalized to lean body mass in female offspring born to dams fed a HFD (Figure 4D), while substrate preference also appeared similar as respiratory exchange ratios were comparable between groups (Figure 4E). The reduced body weight gain in female offspring born to dams fed a HFD is not due to changes in animal ambulatory activity (Figure 4F), but may involve changes in appetite, as 24-hr food intake demonstrated a trend to a mild reduction (Figure 4G).

\section{Discussion}

In this study we observed that female dams fed a HFD for 5 weeks prior to conception and during pregnancy, produced female offspring that exhibited no adverse alterations in glucose homeostasis throughout their juvenile and young adult development. In support of the DOHaD ideology, these female offspring were heavier at weaning, but unexpectedly gained less weight than their counterparts born to dams fed a LFD.

The principle concepts of $\mathrm{DOHaD}$ suggest that the risk for disease susceptibility in a developing individual is actually influenced by a more broader developmental window that includes the period 
171 from pre-conception to oocyte fertilization, versus just the periods of prenatal and postnatal 172 development (Blackmore and Ozanne 2013). As such, we hypothesized that dams fed a HFD prior to 173 conception would produce offspring at increased risk for metabolic syndrome and early onset obesity.

174 To our surprise, female offspring born to dams fed a HFD exhibited no abnormalities in glucose 175 homeostasis. We initially surmised that reasons for this unexpected finding could be due to our 176 experimental model of HFD supplementation in the dam not inducing any overt metabolic dysfunction 177 or adiposity, but assessment of total adiposity in our dams fed a HFD prior to conception revealed 178 robust increases in total fat mass, as well as significant impairments in glucose and insulin tolerance.

179 Taken together, it remains unclear why we did not observe changes in glucose homeostasis in 180 our offspring born to dams fed a HFD prior to conception, and our findings are in contrast to previous 181 studies indicating that offspring born to obese dams are at increased risk for both elevated weight gain 182 and metabolic dysfunction. A study by Howie and colleagues demonstrated that both male and female 183 rat offspring gained weight at increased rates regardless if they were supplemented with a standard 184 chow diet or HFD at weaning if born to dams either fed a HFD (45\% kcal from lard, Research Diets) 185 throughout life, pregnancy, and lactation, or only throughout lactation and pregnancy (Howie et al. 186 2009). Conversely, studies from Laker and colleagues utilizing a pregnancy model in which female 187 C57BL/6 dams were fed a HFD (60\% kcal from lard, Research Diets) for 6 weeks prior to conception 188 and during gestation, demonstrated no differences in offspring body weight over a 1-year period 189 versus offspring born to dams fed a standard chow diet (Laker et al. 2014). Nevertheless, 9-month old 190 offspring born to dams fed a HFD exhibited impairments in glucose and insulin tolerance versus 191 offspring born to dams fed a LFD (Laker et al. 2014). The impairment in glucose homeostasis in the 192 offspring born to dams fed a HFD was attributed to epigenetic changes in PGC1 $\alpha$, a key regulator of 193 mitochondrial function, as PGC1 $\alpha$ methylation was increased and subsequent PGC1 $\alpha$ mRNA 194 expression decreased in skeletal muscles of these offspring. However, our measurements of soleus 195 PGC1 $\alpha$ mRNA expression indicate similar levels at weaning in our female offspring born to dams fed a 
196 HFD, and in 14-week-old female offspring born to dams fed a HFD, versus their female counterpart 197 offspring born to dams fed a LFD (Figure 4H), which may explain why glucose homeostasis was 198 unaltered in our studies. Conversely, it is possible that if we allowed our female offspring to age to 9199 months, we may have also observed impaired glucose homeostasis in the offspring born to dams fed 200 a HFD, supporting previous findings (Laker et al. 2014).

201 Of importance, a number of studies support the notion that epigenetic inheritance may be a 202 critical feature of the DOHaD ideology and the risk for disease susceptibility in a developing individual, 203 including pediatric obesity and metabolic syndrome. For example, dams made obese via liquid 204 enriched diet supplementation (40\% excess calories) for 3 weeks led to robust alterations in offspring 205 DNA methylation of pro-adipogenic factors (e.g. CCAAT enhancer binding protein $\beta$ ) in white adipose 206 tissue stromal vascular cells (Borengasser et al. 2013). To ensure any potential epigenetic alterations 207 in this study were likely acquired via inheritance from the dam fed a HFD and exposure to an obesity208 like intrauterine environment, all offspring born to the dam fed a HFD were cross-fostered to a 209 surrogate lean dam. Conversely, the pregnancy models used by Howie and colleagues or Laker and 210 colleagues had the dams remain on a HFD during the nursing/lactation period. This differentiating 211 factor could influence epigenetic mechanisms controlling body weight gain in the offspring during 212 nursing, potentially explaining the early onset obesity in offspring weaned to a LFD and born to dams 213 fed a HFD observed by Howie and colleagues (Howie et al. 2009), or the impaired glucose tolerance 214 in offspring born to dams fed a HFD observed by Laker and colleagues (Laker et al. 2014). Hence, this 215 may explain why we did not see an increased risk for early onset obesity or metabolic syndrome in our 216 offspring born to dams fed a HFD for 5 weeks prior to conception, as we also cross-fostered our pups 217 to a surrogate dam fed a LFD immediately upon birth. Alternatively, our findings do have a significant 218 limitation in that we did not foster pups born to dams fed a LFD, and it is possible that our 219 observations in female offspring are the result of the stress of fostering, and not the offspring being 220 conceived by a dam fed a HFD and the subsequent intrauterine exposure to an obese metabolic 221 environment. Furthermore, it is possible that maternal care from a foster mother is not as strong as 
222 that from the genetic mother, which could also contribute to our observations in the female offspring. 223 However, we would thus expect to have seen a similar body weight pattern in our male offspring, but 224 body weights in male offspring born to dams fed a HFD and fostered to a dam fed a LFD were 225 identical at all time points when compared to male offspring born to dams fed a LFD without fostering. 226 Nonetheless, this remains a very important lingering question that we plan to address in our future 227 studies.

228 In spite of this limitation, our results are unique as our female offspring born to dams fed a HFD were actually heavier at weaning than their counterparts born to dams fed a LFD, but gained weight at 230 a reduced pace that they actually weighed less once they reached 14-weeks of age. On the other 231 hand, male offspring born to HFD supplemented dams in our study had normal body weights at 232 weaning and gained weight at similar rates to their male offspring counterparts born to LFD 233 supplemented dams. It remains unclear as to why we observed this unique sex-dependent body 234 weight profile in our offspring versus those observed in previously aforementioned studies, though our 235 results in male offspring are consistent with findings in male rat offspring born to HFD supplemented 236 dams by Buckley and colleagues (Buckley et al. 2005). Of interest, our findings are consistent with the 237 set-point theory of body weight regulation (Farias et al. 2011), and it is possible that the reduced rate 238 of body weight gain in female offspring born to a dam supplemented with a HFD could be a centrally 239 regulated defense mechanism resulting from an elevated weaning body weight and intrauterine 240 exposure to an obese environment. As such, it would be interesting to have continually monitored 241 glucose homeostasis in our offspring as they aged even further, and whether this could be negated by 242 weaning the female offspring onto a HFD, though that is beyond the scope of this specific study. It is 243 worth noting though if this set-point theory of body weight regulation is correct, it would have also been 244 anticipated to occur in the male offspring, which we did not observe in our study.

245 Another potential factor is that we utilized a sucrose-matched low-fat control diet for our control 246 dams, whereas most studies simply utilize standard rodent chow. One of the most common standard 247 chow diets employed in animal facilities is the $2018 \mathrm{~S}$ diet of Teklad, and the micronutrient 
248 compositions of these standard chow diets can be vastly different from the micronutrient composition 249 of the Research Diets LFD/HFD utilized in our study. As such, we have observed differences with 250 regards to glucose tolerance and insulin tolerance when comparing 2018S versus Research Diets 251 LFD (unpublished data). This leads to the question of whether obesity itself, or the macronutrient 252 composition of the HFD, is responsible for the offspring phenotypes observed in previous studies, or is 253 it potential differences in micronutrient composition between the Research Diets HFD and the 254 standard chow diet the dam is exposed to that is contributing to the offspring phenotype.

255 In summary, our findings did not reproduce those of previously published papers supporting the $256 \mathrm{DOHaD}$ ideology that obesity and metabolic dysfunction in the pregnant dam lead to increased risk for 257 early onset obesity and metabolic dysfunction in the developing offspring. Whether this is due to 258 differences in micronutrient composition in the diets we utilized versus previous studies, or because 259 we cross-fostered our pups during nursing/lactation to a lean dam fed a LFD, remains to be 260 determined. Future studies characterizing molecular factors that lead to increased body weight at 261 weaning, but reduce the rate of body weight gain in female offspring born to dams fed a HFD are 262 critical.

\section{Acknowledgements}

265 This study was supported by an Innovation Grant from the Women and Children's Health Research 266 Institute, and a New Investigator Operating Grant from the Molly Towell Perinatal Research 267 Foundation. Hanin Aburasayn is supported by a Scholarship from the Saudi Arabian Ministry of Higher 268 Education.

Disclosures

271 The authors have no conflicts to disclose. 


\section{References}

275

276

277

278

279

280

281

282

283

284

285

286

287

288

289

290

291

292

293

294

295

296

297

298

299

300

301

302

303

304

305

306

307

308

309

310

311

312

313

314

315

316

317

318

319

Barker, D.J., Winter, P.D., Osmond, C., Margetts, B., and Simmonds, S.J. 1989. Weight in infancy and death from ischaemic heart disease. Lancet 2(8663): 577-580.

Blackmore, H.L., and Ozanne, S.E. 2013. Maternal diet-induced obesity and offspring cardiovascular health. J Dev Orig Health Dis 4(5): 338-347. doi: 10.1017/S2040174412000761.

Borengasser, S.J., Zhong, Y., Kang, P., Lindsey, F., Ronis, M.J., Badger, T.M., Gomez-Acevedo, H., and Shankar, K. 2013. Maternal obesity enhances white adipose tissue differentiation and alters genome-scale DNA methylation in male rat offspring. Endocrinology 154(11): 4113-4125. doi: 10.1210/en.2012-2255.

Buckley, A.J., Keseru, B., Briody, J., Thompson, M., Ozanne, S.E., and Thompson, C.H. 2005. Altered body composition and metabolism in the male offspring of high fat-fed rats. Metabolism 54(4): 500507. doi: 10.1016/j.metabol.2004.11.003.

Catalano, P.M. 2003. Obesity and pregnancy--the propagation of a viscous cycle? The Journal of clinical endocrinology and metabolism 88(8): 3505-3506. doi: 10.1210/jc.2003-031046.

Farias, M.M., Cuevas, A.M., and Rodriguez, F. 2011. Set-point theory and obesity. Metab Syndr Relat Disord 9(2): 85-89. doi: 10.1089/met.2010.0090.

Flegal, K.M., Carroll, M.D., Kit, B.K., and Ogden, C.L. 2012. Prevalence of obesity and trends in the distribution of body mass index among US adults, 1999-2010. Jama 307(5): 491-497. doi: 10.1001/jama.2012.39.

Holemans, K., Caluwaerts, S., Poston, L., and Van Assche, F.A. 2004. Diet-induced obesity in the rat: a model for gestational diabetes mellitus. Am J Obstet Gynecol 190(3): 858-865. doi: 10.1016/j.ajog.2003.09.025.

Howie, G.J., Sloboda, D.M., Kamal, T., and Vickers, M.H. 2009. Maternal nutritional history predicts obesity in adult offspring independent of postnatal diet. J Physiol 587(Pt 4): 905-915. doi: 10.1113/jphysiol.2008.163477.

Laker, R.C., Lillard, T.S., Okutsu, M., Zhang, M., Hoehn, K.L., Connelly, J.J., and Yan, Z. 2014. Exercise prevents maternal high-fat diet-induced hypermethylation of the Pgc-1alpha gene and agedependent metabolic dysfunction in the offspring. Diabetes 63(5): 1605-1611. doi: 10.2337/db13-1614. Morrison, J.A., Friedman, L.A., and Gray-McGuire, C. 2007. Metabolic syndrome in childhood predicts adult cardiovascular disease 25 years later: the Princeton Lipid Research Clinics Follow-up Study. Pediatrics 120(2): 340-345. doi: 10.1542/peds.2006-1699.

Pereira, T.J., Moyce, B.L., Kereliuk, S.M., and Dolinsky, V.W. 2015. Influence of maternal overnutrition and gestational diabetes on the programming of metabolic health outcomes in the offspring: experimental evidence. Biochem Cell Biol 93(5): 438-451. doi: 10.1139/bcb-2014-0141.

Shankar, K., Harrell, A., Liu, X., Gilchrist, J.M., Ronis, M.J., and Badger, T.M. 2008. Maternal obesity at conception programs obesity in the offspring. American journal of physiology. Regulatory, integrative and comparative physiology 294(2): R528-538. doi: 10.1152/ajpregu.00316.2007.

Ussher, J.R., Baggio, L.L., Campbell, J.E., Mulvihill, E.E., Kim, M., Kabir, M.G., Cao, X., Baranek, B.M., Stoffers, D.A., Seeley, R.J., and Drucker, D.J. 2014. Inactivation of the cardiomyocyte glucagonlike peptide-1 receptor (GLP-1R) unmasks cardiomyocyte-independent GLP-1R-mediated cardioprotection. Mol Metab 3(5): 507-517. doi: 10.1016/j.molmet.2014.04.009.

Ussher, J.R., Fillmore, N., Keung, W., Zhang, L., Mori, J., Sidhu, V.K., Fukushima, A., Gopal, K., Lopaschuk, D.G., Wagg, C.S., Jaswal, J.S., Dyck, J.R., and Lopaschuk, G.D. 2016. Genetic and Pharmacological Inhibition of Malonyl CoA Decarboxylase Does Not Exacerbate Age-Related Insulin Resistance in Mice. Diabetes 65(7): 1883-1891. doi: 10.2337/db15-1145. 


\section{Figure Legends:}

322 Figure 1. 5-Weeks of HFD Supplementation Increases Adiposity and Precipitates Metabolic 323 Dysfunction in Female C57BL/6J Mice. A: Body weights in female C57BL/6J mice fed a LFD or HFD 324 for 5 weeks $(n=6)$. B: Total fat mass in female C57BL/6J mice fed a LFD or HFD for 5 weeks $(n=6)$. 325 C: Fat mass as a percentage of total body weight in female C57BL/6J mice fed a LFD or HFD for 5 326 weeks $(n=6)$. D: Glucose tolerance in female C57BL/6J mice fed a LFD or HFD for 5 weeks $(n=6)$. 327 E: Insulin tolerance in female C57BL/6J mice fed a LFD or HFD for 5 weeks $(n=4)$. Values represent 328 mean \pm SEM. Differences were determined by the use of an unpaired, two-tailed Student's $t$-test, or a 329 two-way ANOVA, followed by a Bonferroni post-hoc analysis. ${ }^{*} P<0.05$.

Figure 2. Body Weights in Male Offspring. Body weights from weaning until 14-weeks of age in male offspring born to C57BL/6J dams fed a LFD or HFD for 5 weeks $(n=3,4)$.

Figure 3. Body Weights and Adiposity in Female Offspring. A: Body weights from weaning until 14weeks of age in female offspring born to C57BL/6J dams fed a LFD or HFD for 5 weeks $(n=6)$. B: Total body weight gain over an 11-week period in female offspring born to C57BL/6J dams fed a LFD or HFD for 5 weeks $(n=6)$. C: Total fat mass and $\%$ fat mass in 4-week old female offspring born to C57BL/6J dams fed a LFD or HFD for 5 weeks $(n=6)$.

D: Total fat mass and \% fat mass in 8-week old female offspring born to C57BL/6J dams fed a LFD or HFD for 5 weeks $(n=6)$. E: Total fat mass and \% fat mass in 14-week old female offspring born to C57BL/6J dams fed a LFD or HFD for 5 weeks $(n=6)$. Values represent mean \pm SEM. Differences were determined by the use of an unpaired, two-tailed Student's $t$-test, or a two-way ANOVA, followed by a Bonferroni post-hoc analysis. ${ }^{*} P<0.05$. 
345 Figure 4. Glucose Homeostasis and In Vivo Metabolism in Female Offspring. A: Oral glucose 346 tolerance in 10-week-old female offspring born to C57BL/6J dams fed a LFD or HFD for 5 weeks $(n=$

347 5). B: Intraperitoneal glucose tolerance in 11-week-old female offspring born to C57BL/6J dams fed a

348 LFD or HFD for 5 weeks $(n=5,6)$. C: Intraperitoneal insulin tolerance in 12-week-old female offspring 349 born to C57BL/6J dams fed a LFD or HFD for 5 weeks ( $n=5,6)$. D: Oxygen consumption in 8-week350 old female offspring born to C57BL/6J dams fed a LFD or HFD for 5 weeks $(n=3)$. E: Respiratory 351 exchange ratio in 8-week-old female offspring born to C57BL/6J dams fed a LFD or HFD for 5 weeks 352 ( $n=3)$. F: Ambulatory activity in 8-week-old female offspring born to C57BL/6J dams fed a LFD or 353 HFD for 5 weeks $(n=3)$. G: 24-hr food intake in 8-week-old female offspring born to C57BL/6J dams 354 fed a LFD or HFD for 5 weeks $(n=3)$. H: Ppargc1a mRNA expression in gastrocnemius muscles from 355 3-week-old or 14-week-old female offspring born to C57BL/6J dams fed a LFD or HFD for 5 weeks (n $356=3,4)$. Values represent mean \pm SEM. Differences were determined by the use of an unpaired, two357 tailed Student's $t$-test, or a one-way ANOVA, followed by a Bonferroni post-hoc analysis. * $P<0.05$. 
A
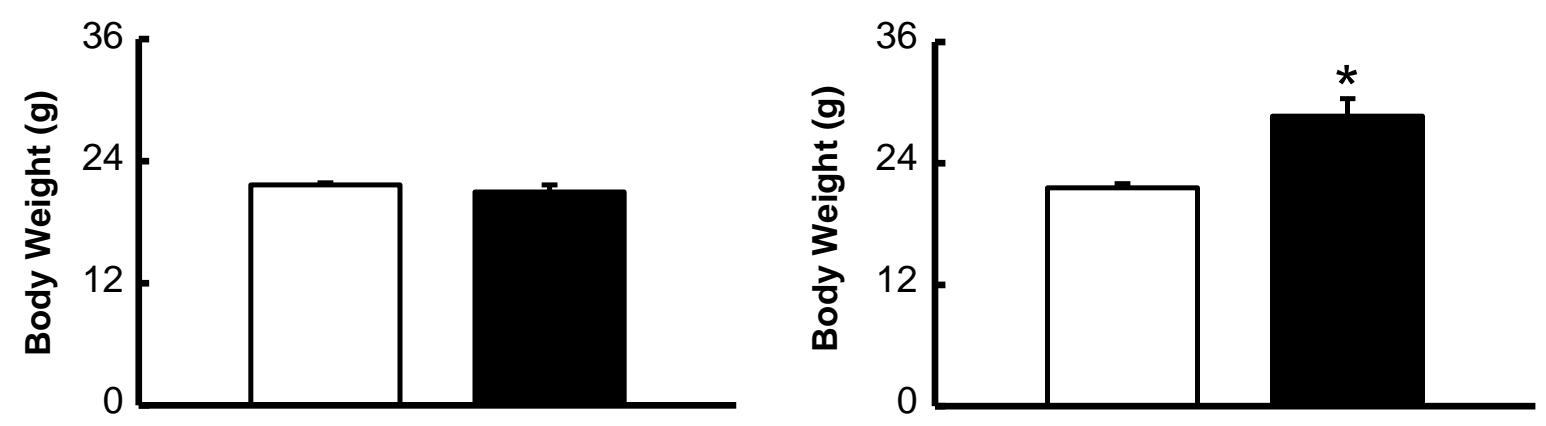

B
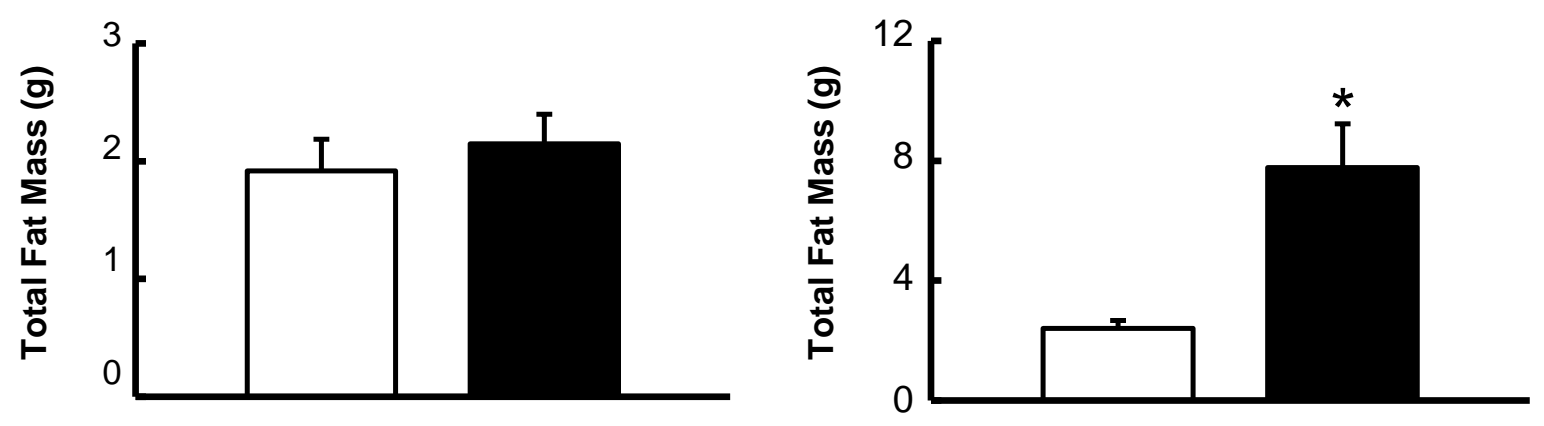

C
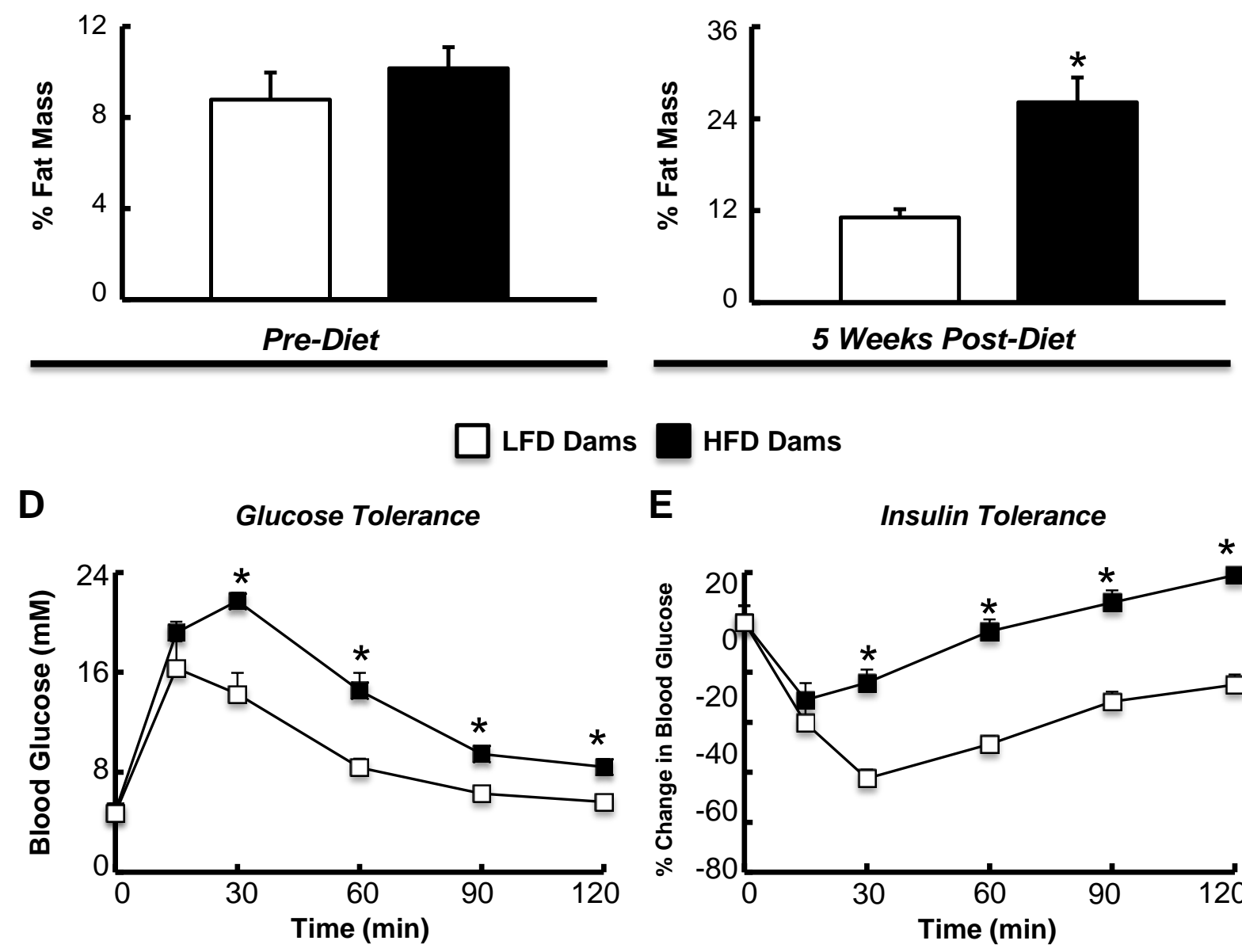

E

Insulin Tolerance

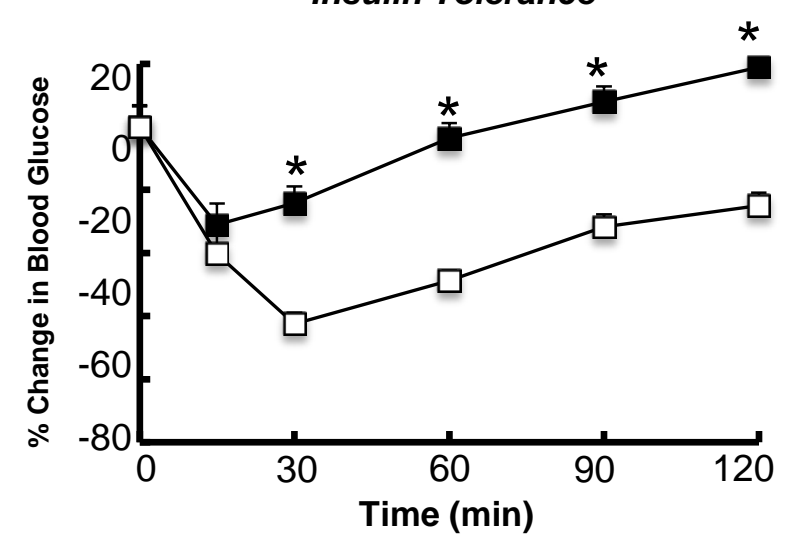


Male Offspring Born to a LFD Dam

Male Offspring Born to a HFD Dam

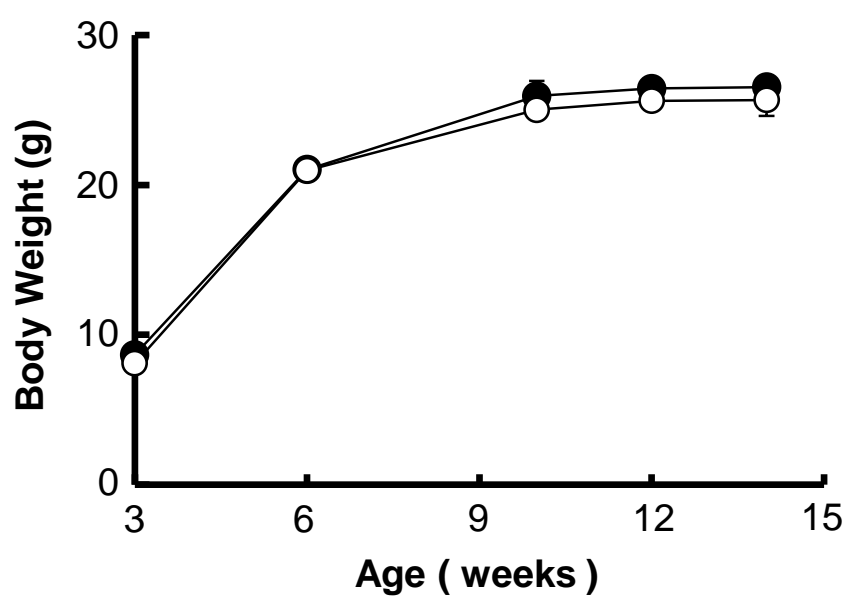

https://mc06.manuscriptcentral.com/cjpp-pubs 
$\square$ Female Offspring Born to a LFD Dam

Female Offspring Born to a HFD Dam

A

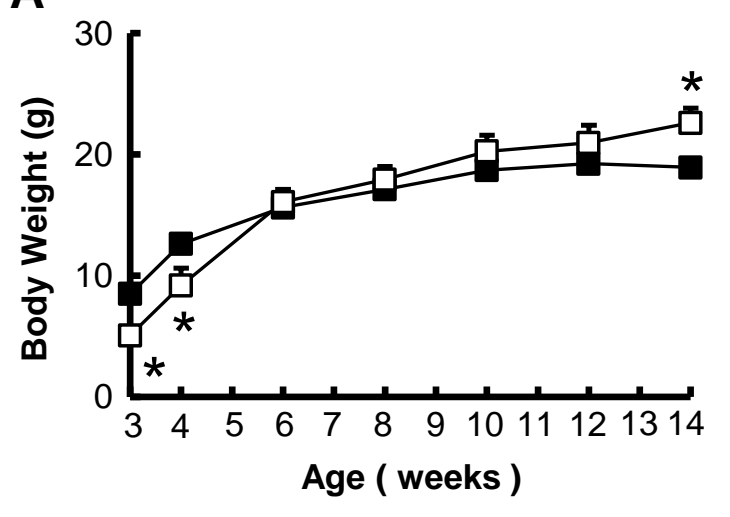

B

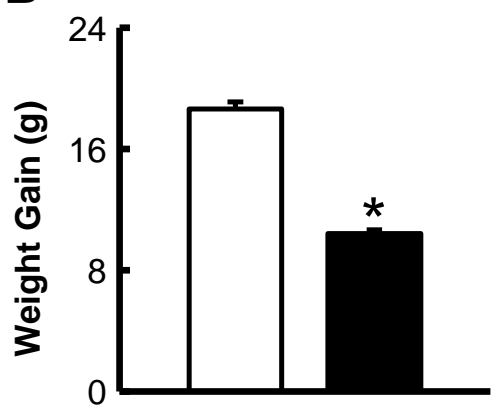

C
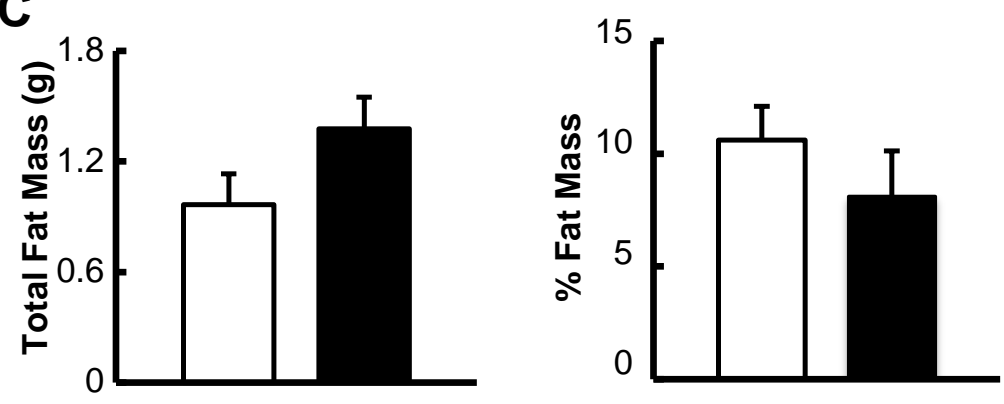

4-weeks of Age

D
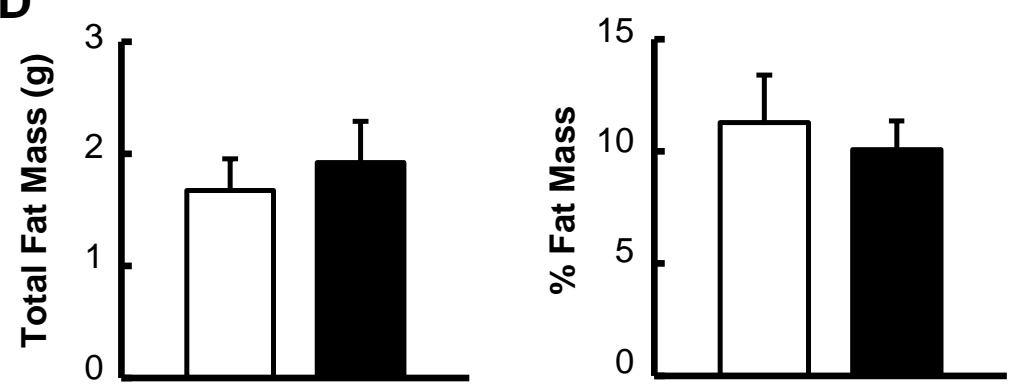

8-weeks of Age

E
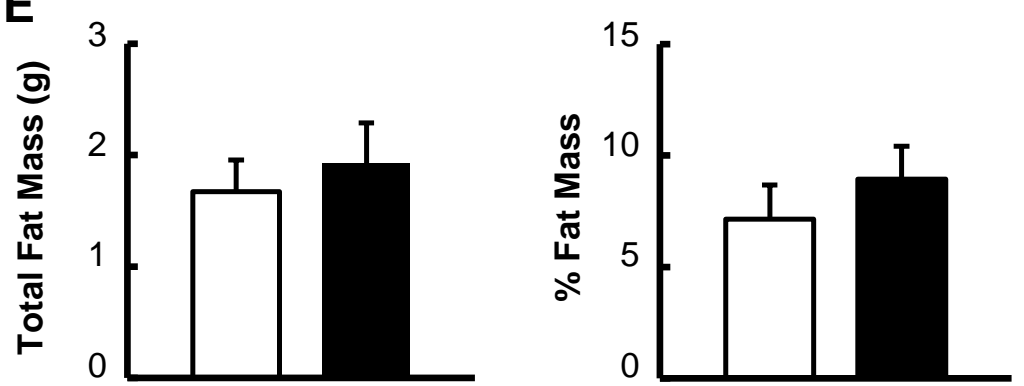

14-weeks of Age 
$\square$ Female Offspring Born to a LFD Dam

Female Offspring Born to a HFD Dam

A

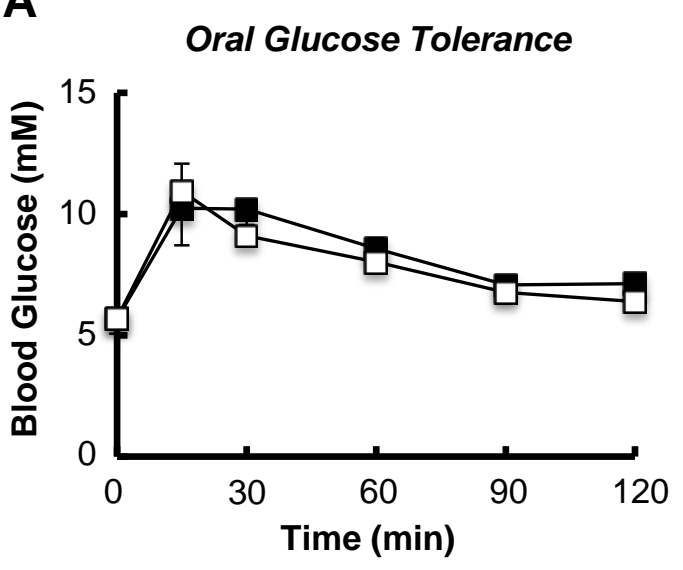

B

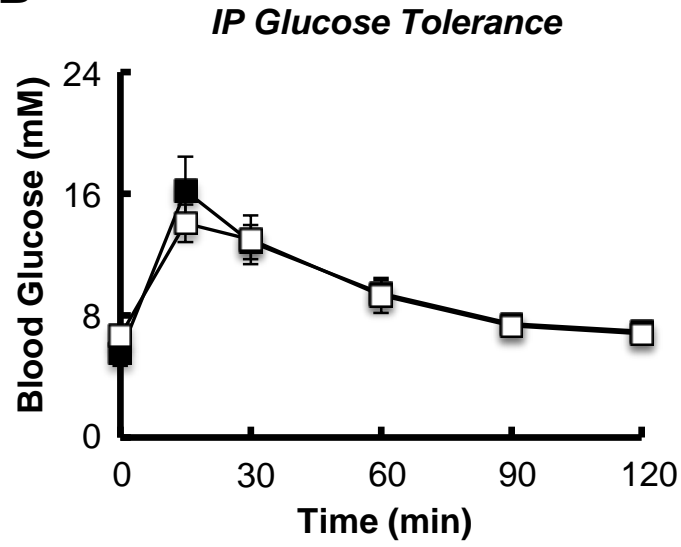

C

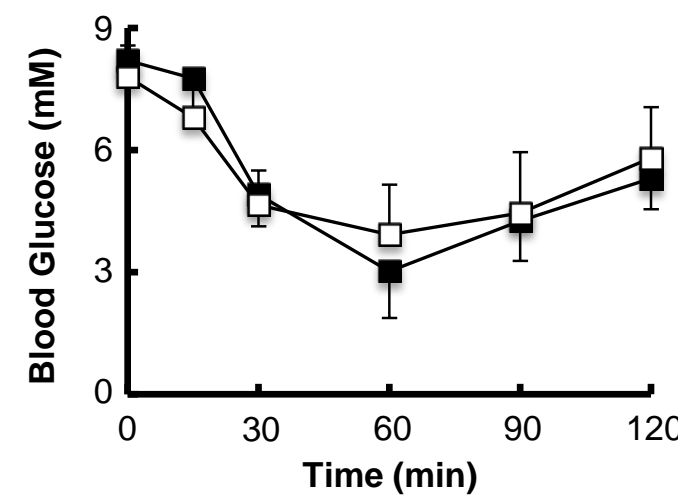

E

F Substrate Preference

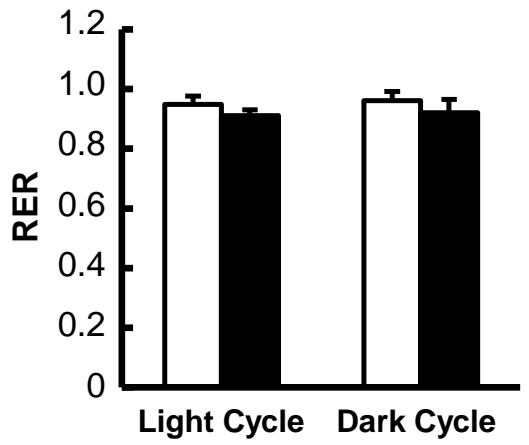

G Ambulatory Activity

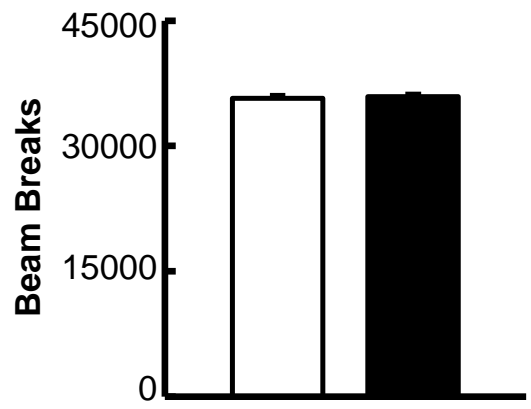

$24 \mathrm{Hr}$ Food Intake

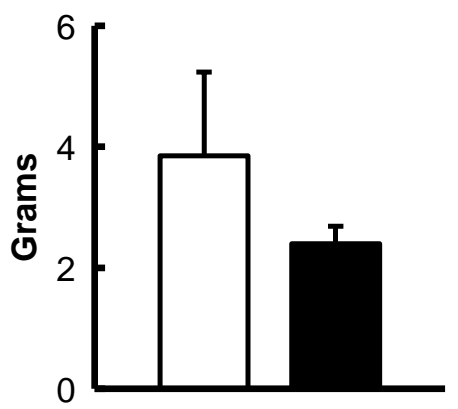

H $\square$ Female Offspring Born to a LFD Dam Female Offspring Born to a HFD Dam

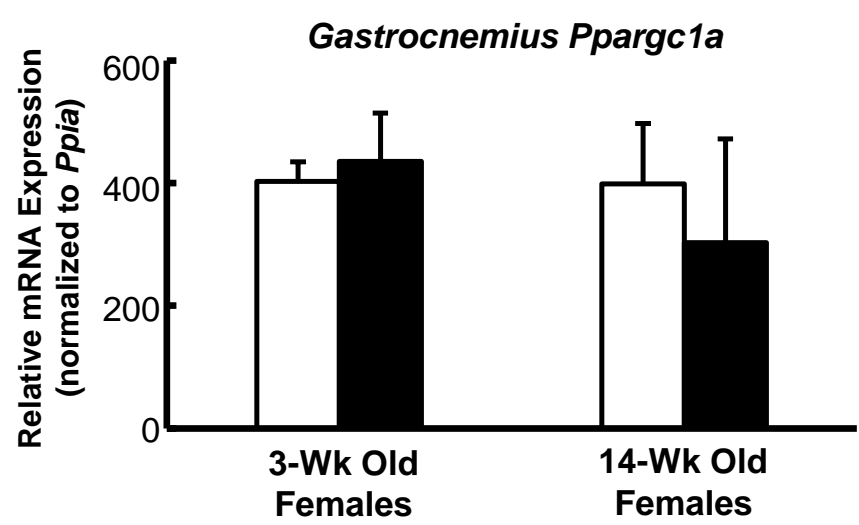

